\title{
Research on the Method of Setting Waiting Area for Non-motor Vehicle at Signal Control Intersection
}

\author{
Yun Xia WANG, Dong Bo LIU \\ Traffic management research institute of the ministry of public security, Wuxi 214151
}

\begin{abstract}
Electric bicycle has become an indispensable important component of the transportation system. The fact is that traffic organization and channelizing design of signal control intersection is not intensive, which cannot adapt to the current traffic demand of non-motor vehicle, such as unclear traffic rules and poor visibility, thus the traffic safety of non-motor vehicle is not optimistic. Therefore, it is necessary to study on traffic organization method based on the demand of non-motor vehicle, which can provide certain theoretical basis for traffic administrative department to make policy and traffic design. This article focuses on the method of setting waiting area for non-motor vehicle at signal control intersection, including the advantages, disadvantages and the applicable conditions.
\end{abstract}

\section{Introduction}

With the rapid development of non-motor vehicle, the traffic order, traffic efficiency and traffic safety at signal control intersection is not optimistic. From the perspective of intersection traffic management, traffic organization and channelization design is extensive, without consideration of the characteristics of electric bicycle. How to give full play to the advantages of electric bicycles, and regulate the traffic order, improve the traffic efficiency and traffic safety level has become a major problem for traffic managers. Therefore, it is necessary to analyze the characteristics of electric bicycle, and propose the method of traffic space optimization, which can adapt to the current non-motor vehicle traffic.

\section{Analysis of traffic characteristics of electric bicycle}

\subsection{Physical Characteristics}

According to the actual measurement data, the average body width of electric bicycles is about $70 \mathrm{~cm}$, and the average body width of bicycles is about $60 \mathrm{~cm}$.

\subsection{Queuing Characteristics}

During the red light, bicycles slow down and queue before the stop line. But electric bicycles tend to staggered together, which is different from motor vehicles. According to the actual observation, it is very common for electric bicycles to line up across the crosswalk.

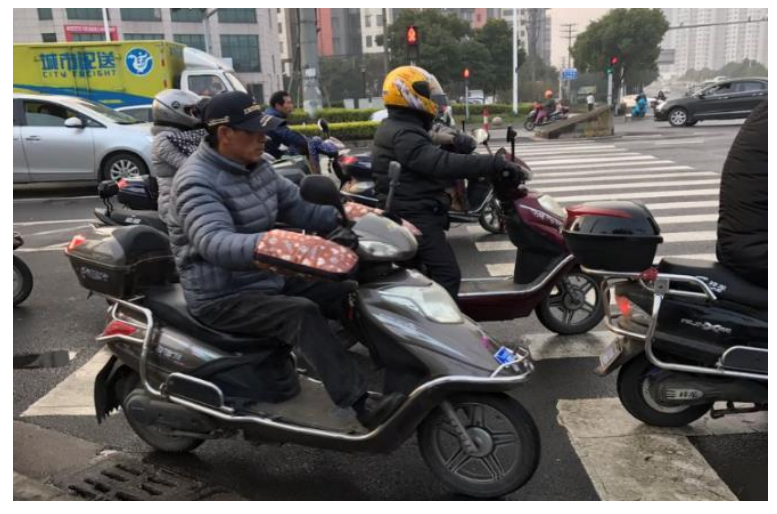

Figure 1 Queue of electric bicycles at signal intersection

\footnotetext{
* Corresponding author: Email: wangyunxiahc@126.com
} 


\subsection{Passing through the Intersection}

Electric bicycle is very flexible, and its instantaneous starting speed is faster than motor vehicles. According to the actual survey, the speed of most electric bicycles through the intersection is relatively close and moderate. The average starting time of the first line of a queue is less than 0.5 seconds. The average speed of straight line electric bicycles is about $14 \mathrm{~km} / \mathrm{h}$, and the average speed of left-turn electric bicycles is about $13 \mathrm{~km} / \mathrm{h}$.
When the signal is green, electric bicycles spread to the intersection gradually just like a fluid. Then they expand to both sides in order to pass through the intersection quickly, taking up the space of motor vehicles and pedestrian. With the gradual increase of electric bicycles, the expansion width increases, forcing the vehicle to slow down, which brings the reduction of traffic capacity as well as the increase of traffic accident. According to the actual survey, the average width of the electric bicycle crossing the street is about 1.4 times compared with the width of the queue.

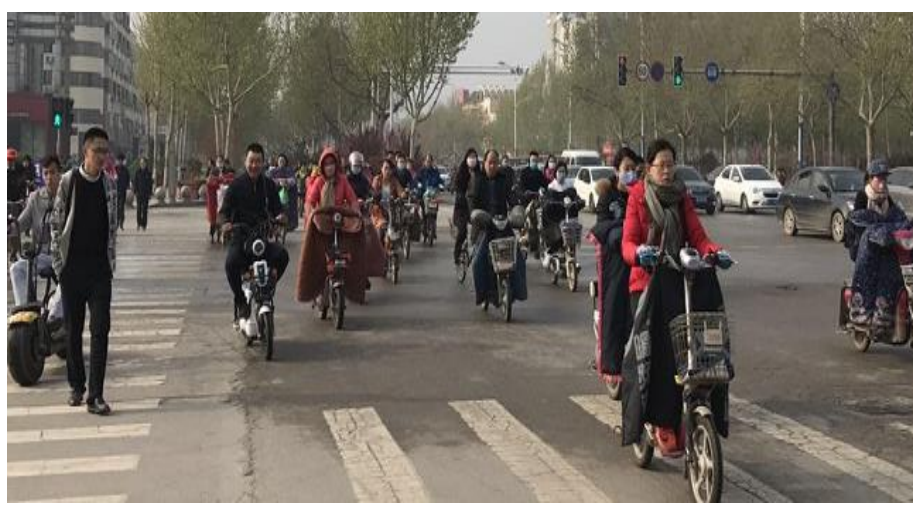

Figure 2 Expansibility of bicycles

\subsection{Traffic Violation Characteristics}

As the electric bicycle is very flexible, start faster than motor vehicle, its illegal behavior at signal control intersection is very common, such as turning off the priority order, do not take non-motor vehicle lanes, reverse driving, and so on.

Based on the traffic characteristics of electric bicycle at signal control intersection, it is practical to study on the traffic organization and management of the non-motor traffic. It is necessary to optimize the design of intersection, the timing of the signal and the supporting management facilities to protect the safety of electric bicycle at signal control intersection, and regulate the traffic order. Based on the characteristics of electric bicycle, the "blank area" which is not used can be used to set waiting space for non-motor vehicle in order to increase its waiting space, to reduce the traffic conflict between motor vehicle and non-motor vehicle, and to improve the intersection of traffic efficiency. According to the location, the waiting space can mainly be divided into the three types as following.

\section{Waiting Area in Front of Stop Line for Non-motor Vehicle}

\subsection{Setting Method}

The stop line of motor vehicle can be stepped back, then the stop line of non-motor vehicle can be set in front. The distance between the motor vehicle and non-motor vehicle stop line is generally determined by the traffic volume of the non-motor vehicles and motor vehicles, as well as the geometry of the intersection, as well as signal control factors. During the red signal phase, the non-motor vehicles are waiting in front of motor vehicles. And once the green signal is turned up, the non-motor vehicles can drive into the intersection immediately, which reduces the interference between main flow non-motor vehicles and motor vehicles. The method of step back of motor vehicle stop line can improve the traffic operation order. In addition, the color pavements (such as green) can be applied to make the non-motor vehicle waiting area more visible[1]. 


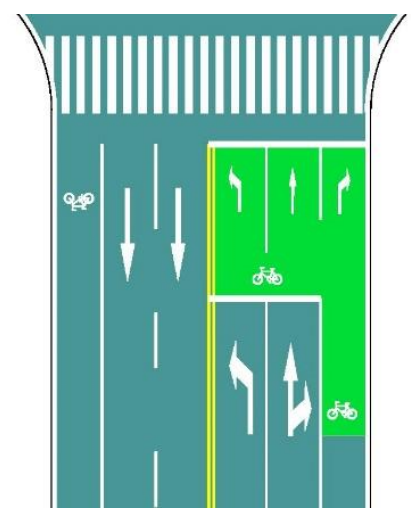

Figure 3 Setting illustration of waiting space in front of motor vehicle stop line for non-motor vehicle

\subsection{Advantage and Disadvantage}

The non-motor vehicle queuing waiting space can be greatly increased, then the efficiency of non-motor vehicles can be improved. While the non-motor vehicles waiting during the red light tend to flock into the intersection. If the traffic volume of non-motor vehicles is large, due to its expansion and lateral movement characteristics, some non-motor vehicles will occupy motorway, which reduces the speed of motor vehicles, as well as the saturated flow and capacity.

\subsection{Applicable Conditions}

This method is fit for the two-phase signal control intersections with 1-2 entrance lanes[2][3]. The successful implement of this method requires enhanced education management of non-motor vehicle drivers, which interpret the reasonable and normative waiting behavior to the drivers. In addition, if the traffic volume of non-motor vehicle is very big, it is required to set specialized left-turn phase for non-motor vehicle in order to reduce the impact of non-motor vehicles on motor vehicles.

\section{4 "Non-signal Control" Waiting Area for Non-motor Vehicle}

With the conditions of high non-motor vehicles volume and enough intersection space, the non-motor vehicle waiting area can be set at the "black area" to reduce the crossing time of non-motor vehicles. The "black area" is the intersection space which is not occupied by motor vehicles. When the high non-motor vehicle volume leads to the interference of normal operation between straight and left-turn flow, the non-motor vehicle waiting area can be divided into straight waiting area and left-turn waiting area. In addition, the color pavements and text makeup can be applied to make the non-motor vehicle waiting area more visible[4].

\subsection{Advantage and Disadvantage}

The non-motor vehicle queuing waiting space can be greatly increased, then the efficiency of non-motor vehicles can be improved. However, when the non-motor vehicles enter the pending area, they are possible to intersect with pedestrians who are crossing the street.

\subsection{Applicable Conditions}

This design is suitable for large intersections. The successful implement of this method requires enhanced education management of non-motor vehicle drivers, which interpret the reasonable and normative waiting behavior to the drivers.

\subsection{Setting Method}

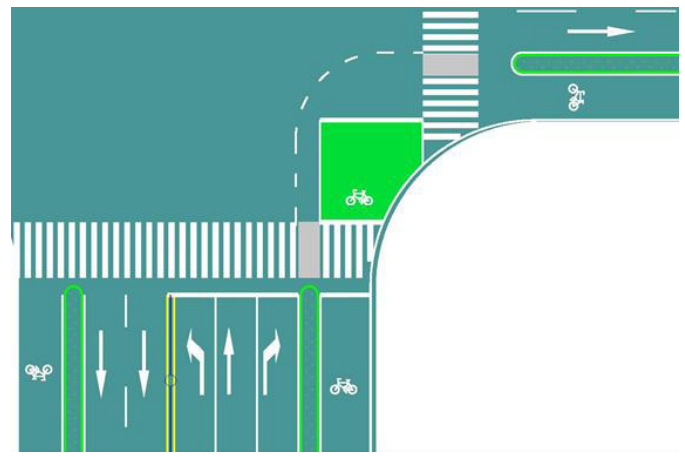

Figure 4 Setting illustration of "Non-light control" Non-motor Vehicle waiting Area 


\section{5 "Signal Control"Waiting Area for Non- motor Vehicle}

\subsection{Setting Method}

With the conditions of high non-motor vehicles volume and enough intersection space, the non-motor vehicle waiting area can be set ahead of crosswalk lines. When the high non-motor vehicle volume leads to the interference of normal operation between straight and left-turn flow, the non-motor vehicle waiting area can be divided into straight waiting area and left-turn waiting area.
It is required to use anti-clockwise unilateral release, when releasing the sraight motor vehicles, the left-turn non-motor vehicles should be prohibited, and when the non-motor vehicles are entering the waiting area, the rightturn motor vehicles should be prohibited. Meanwhile, the LED display screens or traffic signs should be laid out to guide the non-motor vehicles to drive into the waiting areas. For example, when the signal control intersection indicates left-turn phase starts with the end of straight phase, the drivers should be guide into the waiting areas during the left-turn phase of the crossing road. In addition, the color pavements (such as green) can be applied to make the waiting area much more visible.

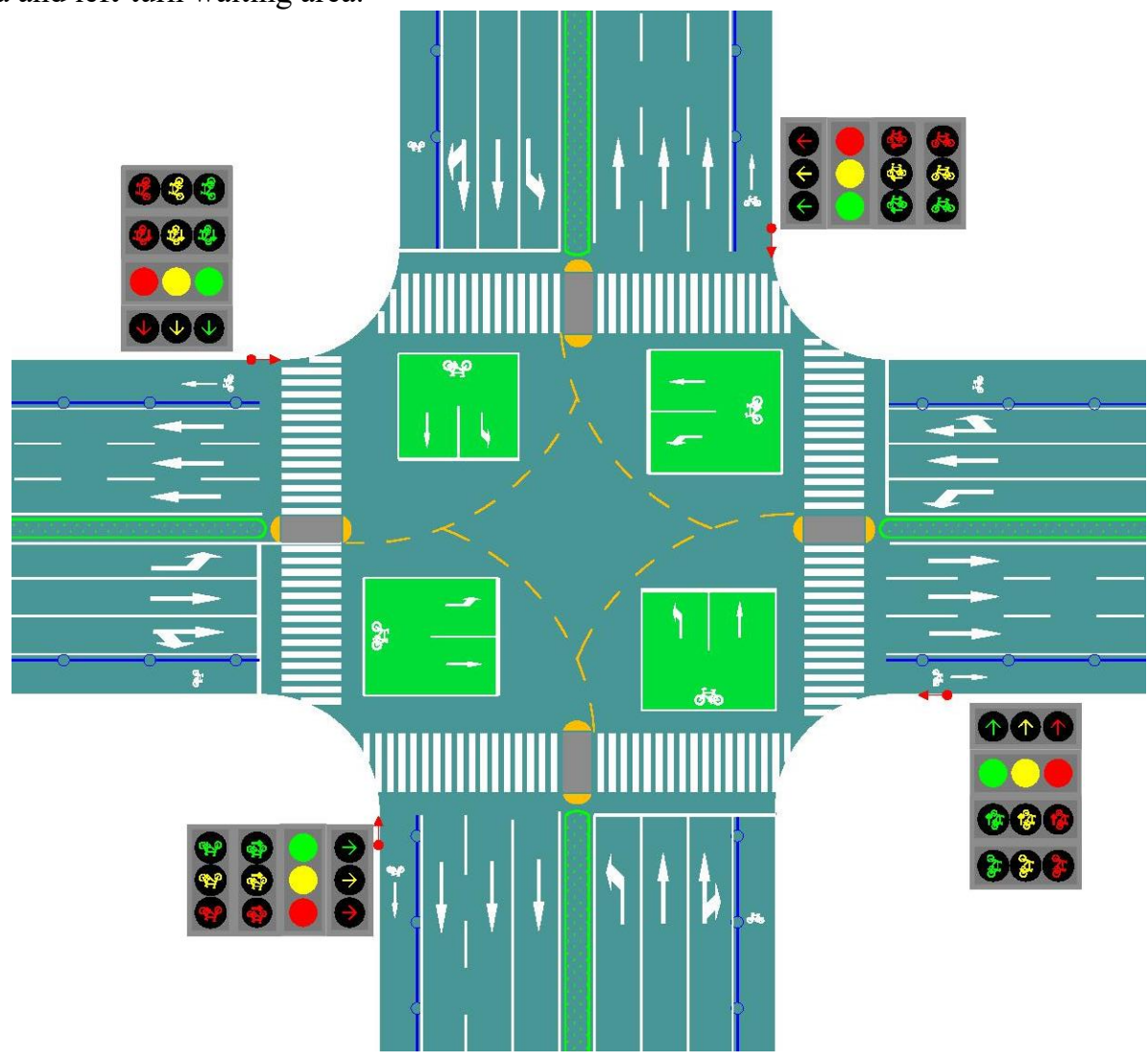

Figure 5 Setting illustration of "light control" Non-motor Vehicle waiting Area

\subsection{Advantage and Disadvantage}

This design makes reasonable use of road resources at signal control intersection, increases the non-motor vehicle queuing waiting area and the green light time, shortens the running cycle time, reduces the intersection signal control delay as well as the conflict between motor vehicle and non-motor vehicle, then the traffic capacity of the intersection is significantly improved. However, it is not easy to implement.

\subsection{Applicable Conditions}

This design is suitable for large intersections (entrance lanes should be at least 3), the size of the area should be able to store the non-motor vehicles. At the same time, it requires non- motorcyclists to take the initiative to comply with the rules.

\section{Typical Case Analysis}

\subsection{Channelization State and Existing Problem}

The case intersection is a very important intersection, which connects the east and west district of the city. The traffic volume of non-motor vehicle is very large, and there is serious conflict between motor vehicles and nonmotor vehicles which seriously affects the traffic efficiency of the intersection. To improve the situation, the intersection "blank area" is used to set the "signal control" waiting area for non-motor vehicle, with four directions 
unilateral clockwise release to eliminate the traffic conflict. Specific measures are shown in Fig.5.

\subsection{Analysis of Program Implementation Effect}

Before the improvement, the motor vehicles and nonmotor vehicles are in the same signal control phase, and non-motor vehicles often occupy the motorway, which leads to serious traffic safety risks. After the improvement, on one hand, road space resources are made full use, and non-motor vehicles are concentrated in the waiting area to be released before motor vehicles, which brings the reduction of the traffic conflict and regulates traffic order of the intersection; on the other hand, motor vehicles and non-motor vehicle traffic efficiency has been improved significantly, for example, both morning and evening peak motor vehicle capacity are increased by about $7 \%$.

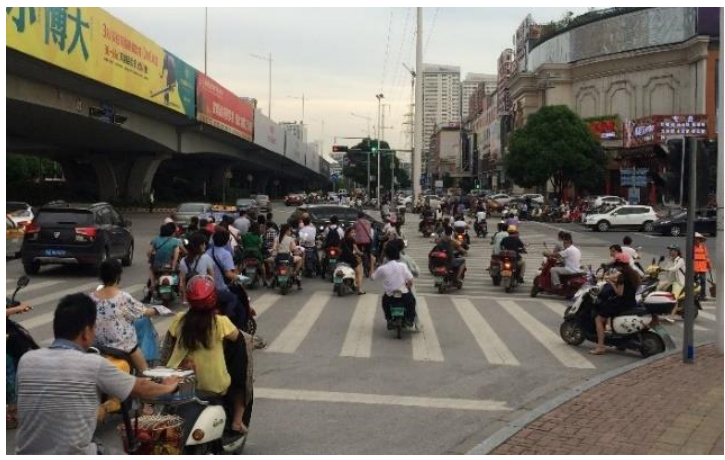

Figure 6 Before the Improvement

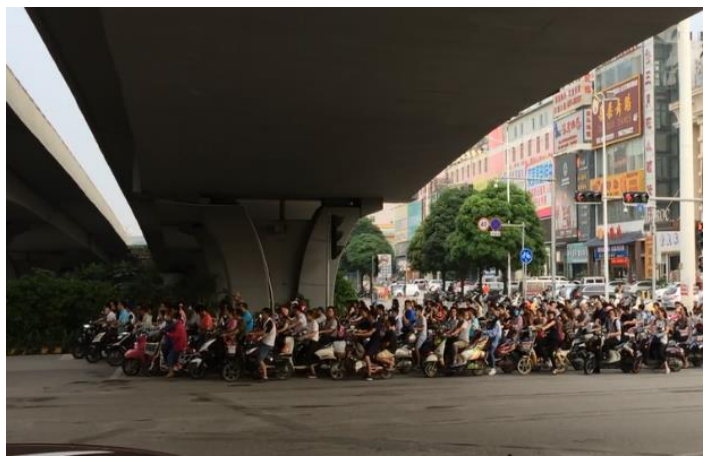

Figure 7 After the Improvement

\section{Conclusions}

Based on the analysis of typical traffic characteristics of non-motor vehicles, the space optimization method for non-motor vehicles of typical signalized intersections is proposed in this research, which aims to support the traffic channelization design of signal controlled intersections and improve the operation environment of non-motor vehicles. The future research will focus on the space and time integrated optimization method which fit for the operation demand of non-motor vehicle crossing.

\section{Acknowledgement}

The authors thanks for the sponsor of National Science and Technology Support Program with Grant No. 2014BAG03B01.

\section{References}

1. Junjie Lu. Technology and application of colored pavement $[\mathrm{J}]$. Fujian Construction Science \& Technology, 2013, (5): 65-67.

2. Xiaoguang Yang. Traffic Design [M]. Beijing: China Communications Press, 2010.

3. Xiaoguang Yang. Manual of urban traffic design [M]. Beijing: China Communications Press, 2003.

4. National standards "Road traffic signs and markingsPart3: Road traffic markings" (GB5768.3-2009) 